\title{
Cirurgia por Orifícios Naturais Transcolônica: Acesso NOTES Peri-retal (PNA) para Excisão Mesoretal Total
}

\author{
Transcolonic Natural Orifice Surgery: Peri-rectal NOTES Access (PNA) \\ for Total Mesorectal Excision
}

\author{
RICARDOZORRON $^{1}$; DJALMACOELHO ${ }^{2}$; LUCIANAFLACH $^{3} ;$ FABIANO BATISTALEMOS $^{4}$; \\ MOACYR SIMAS MOREIRA ${ }^{5} ;$ PRISCILASARAIVA OLIVEIRA $^{6} ;$ ALAIN MARCEL BARBOSA $^{7}$
}

\begin{abstract}
${ }^{1}$ Professor e Chefe do Departamento de Cirurgia, HCTCO- FESO, Teresópolis, Rio de Janeiro- Brasil. Serviço de Cirurgia - Hospital Municipal Lourenço Jorge, Rio de Janeiro- Brasil; ${ }^{2}$ Serviço de Cirurgia - Hospital Municipal Lourenço Jorge, Rio de Janeiro- Brasil. Universidade Estacio de Sa, Rio de Janeiro- Brasil; ${ }^{3}$ Serviço de Cirurgia Hospital Municipal Lourenço Jorge, Rio de Janeiro- Brasil; ${ }^{4}$ Serviço de Cirurgia - Hospital Municipal Lourenço Jorge, Rio de Janeiro-Brasil; ${ }^{5}$ Serviço de Cirurgia - Hospital Municipal Lourenço Jorge, Rio de Janeiro- Brasil; ${ }^{6}$ Serviço de

Cirurgia - Hospital Municipal Lourenço Jorge, Rio de Janeiro- Brasil; ${ }^{7}$ Serviço de Cirurgia - Hospital Municipal Lourenço Jorge, Rio de Janeiro- Brasil.
\end{abstract}

ZORRON R; COELHO D; FLACH L; LEMOS FB; MOREIRA MS; OLIVEIRA PS; BARBOSA AM. Cirurgia por Orifícios Naturais Transcolônica: Acesso NOTES Peri-retal (PNA) para Excisão Mesoretal Total. Rev bras Coloproct, 2010;30(1): 014-022.

RESUMO: Objetivos: Cirurgia por orifícios naturais tem sido recentemente aplicada em series clínicas para cirurgia abdominal. Apesar de potenciais vantagens do acesso NOTES transcolônico para doenças colorretais, este ainda não havia sido utilizado clinicamente. O presente trabalho descreve a primeira aplicação bem-sucedida de NOTES transcolônico da literatura, em uma nova abordagem de excisão mesoretal total (TME) para cancer de reto. Métodos: Foi obtida aprovação de Comitê de Ética em Pesquisa para cirurgias por orifícios naturais, e o paciente assinou termo de consentimento informado. Em um paciente de 54 anos portador de adenocarcinoma de reto, o procedimento de retossigmoidectomia e linfadenectomia, com excisão mesoretal total foi realizada utilizando um acesso posterior transcolônico pouco acima da borda anal. A dissecção mesorretal foi conseguida utilizando um colonoscópio flexível e instrumentos endoscópicos, com assistência laparoscópica. O espécime foi retirado via transanal, e anastomose foi transorificial, com estoma proximal de proteção. Resultados: O tempo operatório foi de $350 \mathrm{~min}$, não ocorrendo complicações operatórias. A evolução pósoperatória foi favorável, e o paciente recebeu alta no sexto dia de pós-operatório com dieta plena. Conclusão: Este primeiro relato bem sucedido de cirurgia NOTES transcolônica traz potencialmente novas fronteiras de aplicações clínicas na cirurgia minimamente invasiva. O tratamento de doenças colorretais utilizando o novo acesso flexível PNA (Perirectal NOTES Access) é uma promissora nova abordagem, paralelamente à laparoscopia e cirurgia aberta, para melhoria do tratamento dos pacientes.

Descritores: Câncer do reto; excisão mesorretal total; cirurgia colorretal; TME; Cirurgia por Orificios Naturais; NOTES; Acesso transcolonico; PNA; laparoscopia.

\section{INTRODUÇÃO}

A cirurgia através de orifícios naturais (NOTES-Natural Orifice translumenal Endoscopic Surgery) surgiu nos anos recentes como uma promissora alter- nativa minimamente invasiva à cirugia aberta e laparoscópica para tratamento de afecções abdominais. Benefícios potenciais em evitar complicações da ferida cirúrgica, como hérnias, infecção, obstrução intestinal, além do benefício cosmético, levaram às primeiras

Trabalho realizado no Serviço de Cirurgia, Hospital Municipal Lourenco Jorge, Rio de Janeiro, Brasil.

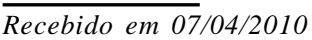

Aceito para publicação em 07/04/2010 
series de aplicações clínicas da literatura para NOTES por acesso transvaginal e transgástrico ${ }^{1,2}$. Inicialmente, foram realizados procedimentos mais simples como colecistectomia transvaginal e transgástrica, apendicectomia e estadiamento de neoplasias.

Cirurgias utilizando o acesso colônico para realização de cirurgia abdominal (NOTES transcolônico) tem sido objeto de pesquisa de pesquisa experimental recente, sugerindo que o acesso poderia ser uma opção atraente para tratamento de afecções colorretais e abdominais ${ }^{3-8}$. Obstáculos técnicos iniciais como o risco de infecção e fistula, acesso seguro à cavidade $\mathrm{e}$ fechamento confiável do orifício colônico, que permaneceram como problemas que retardaram o uso do acesso, comparado com os acessos transvaginal e transoral. O grupo de pesquisa em nossa instituição, desenvolvendo técnicas NOTES transcolônicas pioneiras em modelo animal, estabeleceu a técnica Transcolonica Periretal para ganhar acesso e terapia na cavidade abdominal e espaço retroperitoneal. A técnica de PNA (Perirectal NOTES Access) utilizando um endoscópio flexível através de uma incisão retal baixa, com facilidade de acesso e fechamento e pode ter indicação para uma vasta gama de procedimentos minimamente invasivos.

Excisão Mesoretal Total (TME) com linfadenectomia tornou-se o tratamento padrão na ressecção curativa do cancer de reto ${ }^{9,10}$, e os resultados da cirurgia laparoscópica tem demonstrado evidências de ser similar à cirurgia aberta em estudos recentes ${ }^{11}$. A técnica desenvolvida neste presente trabalho em nossa instituição e testada em modelo animal (Perirectal NOTES Access- PNA), foi utilizada para iniciar aplicações clínicas. O estudo descreve o primeiro procedimento NOTES via transcolônica da literatura mundial, utilizando instrumentos flexíveis em um caso de retossigmoidectomia e excisão mesoretal total para cancer de reto.

\section{MÉTODOS}

O estudo foi aprovado pela Comissão de Ética em Pesquisa (CEP-SMS Rio de Janeiro) para trials clínicos em NOTES, e todos os métodos estão em acordo com os critérios éticos da Declaração de Helsínqui da WMA(World Medical Association), revisão 1989 e as diretrizes para a saúde do National Institute of Health, EUA, e da resolução n 196/96 do Ministério da Saúde, Brasil. O paciente foi informado sobre as possibili- dades, vantagens e desvantagens da cirurgia, e complicações, e assinou Termo de Consentimento Livre e Esclarecido (TCLE). O grupo de pesquisa consistiu de um time multidisciplinar, e Hospital Verinário foi disponibilizado para testar as alternativas terapêuticas em modelo animal com estudos de sobrevida. Os estudos em modelo suíno foram realizados durante 10 meses, com resolução dos problemas de orientação espacial, insuflação, e evolução de instrumental.

Um paciente do sexo masculino com indicação cirúrgica oncológica para câncer de reto foi submetido a retossigmoidectomia, linfadenectomia e excisão mesorretal total (TME) flexível através de acesso NOTES transcolônico com assistência laparoscópica (Figura 1), e parâmetros intra e pós-operatórios foram prospectivamente documentados. A equipe cirúrgica foi composta por um cirurgião, um gastroenterologista-endoscopista e dois residentes.

Em um paciente de 54 anos que apresentava sangramento retal e perda de peso de $12 \mathrm{~kg}$ em 10 meses, foi feito o diagnóstico de adenocarcinoma de reto a $8 \mathrm{~cm}$ da borda anal, confirmado por colonoscopia

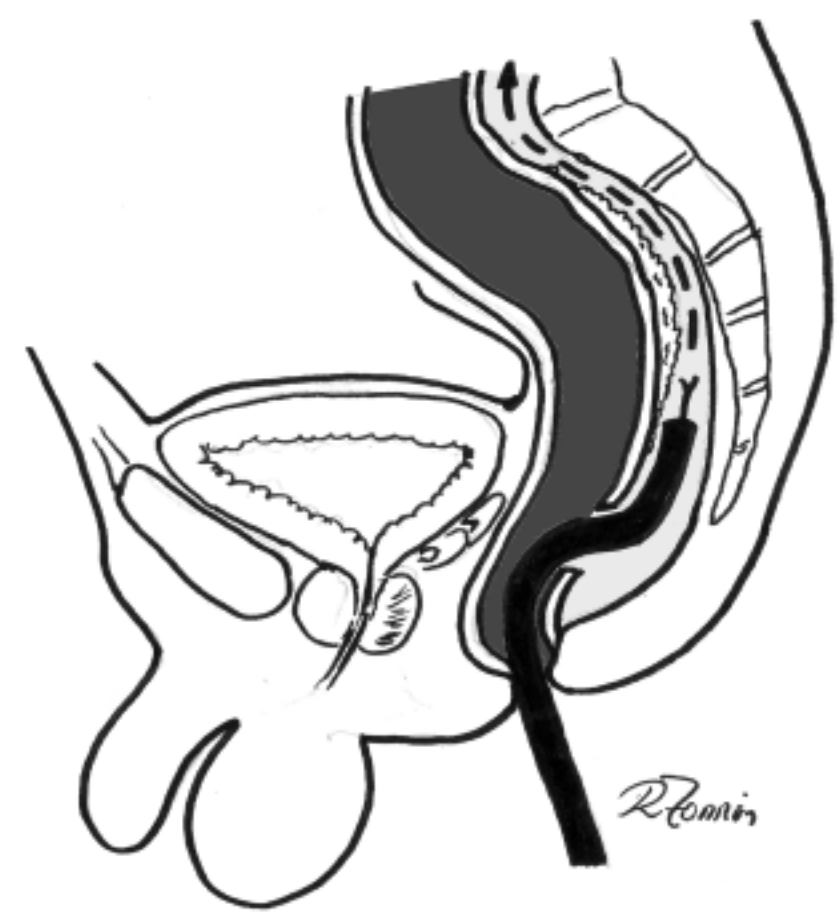

Figura 1 - Visão esquemática do acesso NOTES perirretal (Perirectal NOTES Access-PNA) para escisão mesorretal total para câncer retal. O endoscópio é inserido diretamente no espaço présacral, $3 \mathrm{~cm}$ acima da linha denteada, possibilitando cirurgia flexível no plano anatômico de dissecção iniciando inferiormente em direção proximal. 
e retossigmoidoscopia rígica com biópsia. Em virtude de estenose de $90 \%$ do lumen pelo tumor (Figura 2A), a colonoscopia não foi capaz de transpassar a estenose e avaliar o colon proximal, e não foi realizado preparo de cólon pré-operatório pelo mesmo motivo. Radioquimioterapia neoadjuvante não foi indicada em virtude do padrão obstrutivo da lesão. O paciente não tinha co-morbidades, e foi classificado com risco operatório ASA I. Tomografia computadorizada pré-operatória demonstrou limitação do tumor de $4 \mathrm{~cm}$ de diâmetro ao reto, e sem evidências de metástases peritoneais ou hepáticas. Dosagem de CEA (antígeno carcinoembrionário) estava dentro dos limites da normalidade $(2.8 \mathrm{mg} / \mathrm{dl})$. Esta primeira aplicação clínica mundial de NOTES transcolônico foi realizada em 13.11.2009.

\section{Técnica Cirúrgica}

O paciente foi instalado em posição de LloydDavies sob anestesia geral. Profilaxia antibiótica foi realizada na indução anestésica com $400 \mathrm{mg}$ de ciprofloxacina and 500mg de metronidazol. O equipamento utilizado foi um videocolonoscópio Olympus 130 de canal simples (Olympus, Japão) e um set laparoscópico (KarlStorz, Alemanha). Um espéculo anal foi inserido via transanal, e o reto foi fechado por uma sutura circunferencial em bolsa de Vicryl 2.0 sob visão direta para evitar contaminação pelo cólon proximal, antes de iniciar o acesso NOTES. A sutura foi colocada a $4 \mathrm{~cm}$ da borda anal, e assim foi planejada uma incisão posterior no coto retal a $3 \mathrm{~cm}$ da borda anal. Após o fechamento do limite da ressecção retal, foi realizada desinfecção do reto distal utilizando irrigação com solução de iodopovidine degermante.

Devido aos princípios oncológicos de ligadura prévia alta da artéria mesentérica inferior antes da manipulação próxima ao tumor, uma câmera laparoscópica de $30^{\circ}$ foi inserida via transumbilical, e dupla ligadura da IMA foi realizada com Prolene 2-0 ao nível de sua origem utilizando técnica de três trocartes antes da Excisão Mesoretal Total (TME). Passando para a abordagem transanal, a linha denteada foi identificada, e uma incisão posterior no reto de aproximadamente $2.5 \mathrm{~cm}$ foi realizada exatamente na programada linha de ressecção retal utilizando cautério monopolar sob visão direta. $\mathrm{O}$ orificio de acesso ao espaço pré-sacral foi testado por inspeção digital e o colonoscópio foi inserido diretamente no espaço perirretal retroperitoneal. (Figura 2B-2D). O acesso
NOTES Perirretal (PNA) implica em uma perfuração baixa posterior ao plano anatômico entre a fascia présacral e a fascia propria, evoluindo com uma dissecção proximal ascendente e circunferencial (Figura 1). Dissecção através da insuflação de $\mathrm{CO} 2$ e pela extremidade do endoscópio foi iniciada, mantendo a fascia pré-sacral como orientação. Excisão mesoretal total foi progressivamente obtida utilizando nova tecnologia em tesoura endoscópica monopolar (ApolloEndosurgery, Austin, USA) através do plano oncológico ideal de dissecção.

A progressão da dissecção TME para os aspectos lateral e anterior também foi realizada utilizando tesoura endoscópica flexível e dissecção com o endoscópio (Figura 2C), e a cavidade abdominal foi alcançada pela abordagem NOTES no aspecto lateral da dissecção. Neste momento, houve perda do retropneumoperitônio devido a vazamento para a cavidade abdominal. Assistência laparoscópica foi então utilizada para dissecção e liberação do ângulo esplênico do cólon, e para completar a dissecção do sigmóide e liberação do aspecto superior do reto.

O espécime foi apreendido por meio de pinça Duval intraretal, e o cólon foi extraído totalmente por via transanal (Figura 3A). A secção do cólon exposto foi realizada por incisão monopolar, e o limite do cólon proximal foi preparado inserindo a ogiva do grampeador circular com sutura de Prolene 2.0 (Figura 3B). O cólon proximal com a ogiva foi reinserido pelo ânus para a cavidade. $O$ coto retal foi seccionado via transanal em toda sua circunferência, e uma sutura em bolsa do coto retal foi realizada com Prolene 2.0. para possibilitar a introdução retal do grampeador circular 28 e anastomose (Figura 3C). Colostomia proximal em alça à dreita do cólon transverso foi realizada para proteção da anastomose. Drenagem fechada da dissecção pélvica foi instalada com dreno tubular à vácuo. $\mathrm{O}$ espécime ressecado demonstrou ressecção mesorretal adeuqda com preservação do envelope mesorretal, e uma margem distal de $4 \mathrm{~cm}$ abaixo do limite tumoral.

\section{RESULTADOS}

O tempo operatório foi de $350 \mathrm{~min}$, e tempo de anestesi de 410 min. Comparados aos achados préoperatórios, nível de hemoglobina e hematócrito $(11.9 \mathrm{mg} / \mathrm{dl}$ e $35.3 \%)$ tiveram pouca modificação após o procedimento no primeiro dia de pós-operatório $(11.1 \mathrm{mg} / \mathrm{dl}$ e $32.3 \%$ respectivamente). Apesar do tem- 

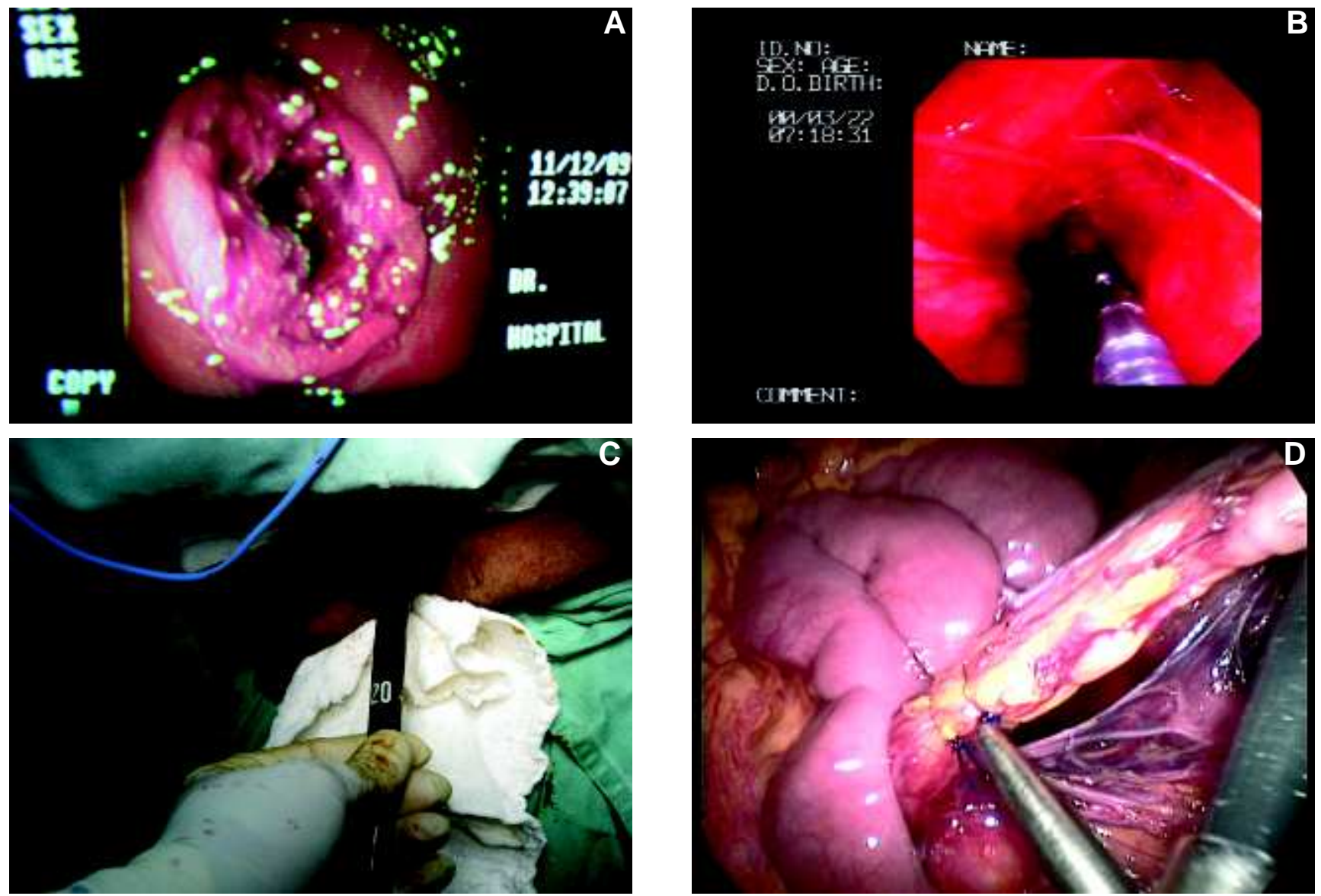

Figura 2 - A. Colonoscopia pré-operatória demonstrando estenose de $90 \%$ do lumen pelo tumor. B. O colonoscópio é inserido diretamente no espaço perirretal retroperitoneal através de incisão posterior no reto, e evolui paralelamente ao lumen retal. C. Excisão mesorretal total (TME) é progressivamente conseguida utilizando tesoura endoscópica monopolar dentro do plano oncológico ideal ("holy plane") Progressão da dissecção TME para os aspectos laterais e anterior, alcançando a cavidade abdominal. D. Ligadura e secção laparoscópica da artéria mesentérica inferior ao nível da aorta utilizando ligaduras com Prolene 2.0.

po operatório, perda sangüínea intra-operatória estimada foi pequena $(60 \mathrm{ml})$.

O paciente teve uma boa recuperação pós-operatória, e iniciou movimentos peristáticos e dieta oral líquida no terceiro dia de pós-operatório. Antibioticoterapia foi continuada devido ao preparo inadequdo do cólon e contaminação local do coto retal. O paciente queixou-se de parestesia transitória de ambos os pés, possivelmente devido ao posicionamento operatório, que desapareceu após 10 dias. Escala visual analógica de dor demonstrou um nível zero de dor duas horas após o procedimento, nível zero na manhã do $1^{\circ} \mathrm{DPO}$, e nível 3 no $7^{\circ}$ DPO. O aspecto externo do abdome demonstrou bom resultado evitando a incisão habitual para extração do espécime (Figura 3D). O paciente recebeu alta no $6^{\circ}$ DPO, e não houve complicações infecciosas ou gerais em revisão no $30^{\circ}$ dia de pós-operatório.

\section{DISCUSSÃO}

Após a evolução da videocirurgia nos anos recentes, a comunidade cirúrgica demonstrou crescente interesse em procedimentos ainda menos invasivos para diagnóstico e terapia de afecções abdominais ${ }^{12}$. Apesar do entusiasmo inicial e das promissores benefícios acerca das técnicas NOTES, elas ainda não sinalizaram superioridade em nenhuma indicação clínica ainda sobre as técnicas existentes ${ }^{1,2}$. Estudos clínicos e experimentais ainda demonstram que evolução tecnológica é necessária para expandir as possibilidades deste novo campo, possibilitando segurança e eficiência às tecnicas NOTES.

Publicações recentes e apresentações em eventos científicos desde 2007 descreveram as aplicações clínicas iniciais do novo método, após aprovação 

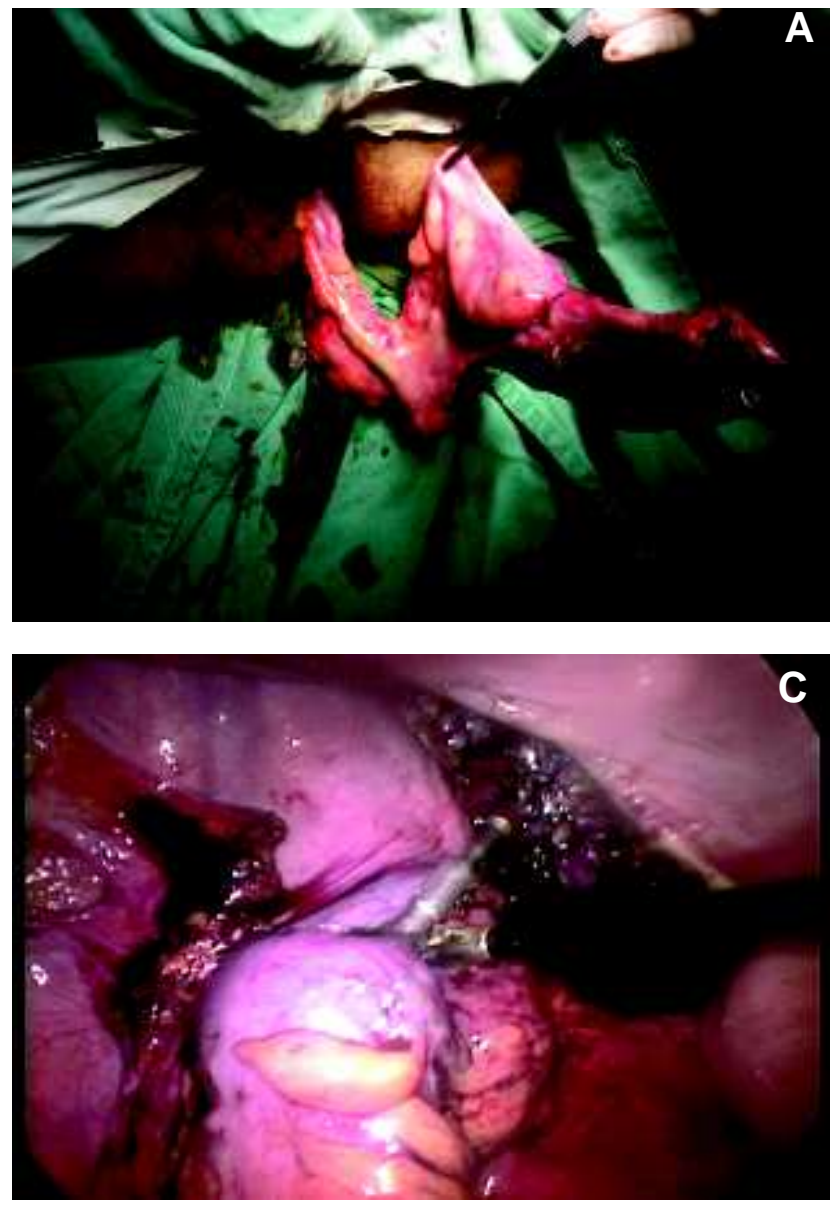

Figura 3 - A. Extração transanal completa do espécime com intacto envelope da excisão mesorretal. B. Resseção transanal do retossigmóide mobilizado e preparação do coto proximal com ogiva do grampeador circular. $\boldsymbol{C}$. Anastomose circular grampeada. $\boldsymbol{D}$. Aspecto externo do abdome no $10^{\circ} \mathrm{DPO}$.

dos trials em Comitês de Ética e Pesquisa clínica em cirurgia por orificios naturais em diferentes países. Até o momento, foram publicados 195 casos humanos mundialmente, e ainda outros 362 casos incluídos no estudo multicêntrico internacional IMTN NOTES Multicenter Study $^{13}$, na maior parte descrevendo baixas taxas de complicações. Desde que Zorron e cols, Marescaux e cols, Zornig e cols, e Branco e cols descreveram bemsucedidas colecistectomis NOTES por via transvaginal em $2007^{14-17}$, outros grupos seguiram e publicaram seus resultados clínicos iniciais das técnicas, na maior parte com assistencia laparoscópica para propósito de retração ou visualização ${ }^{18-28}$. Apendicectomia transgástrica foi também apresentada clinicamente em congressos por Rao e Reddy utilizando material endoscópico disponível desde $2005{ }^{29}$, e recuperação bem-sucedida de gastrostomia foi descrita por Marks e cols ${ }^{30}$. Apendicectomia NOTES transvaginal foi ini-
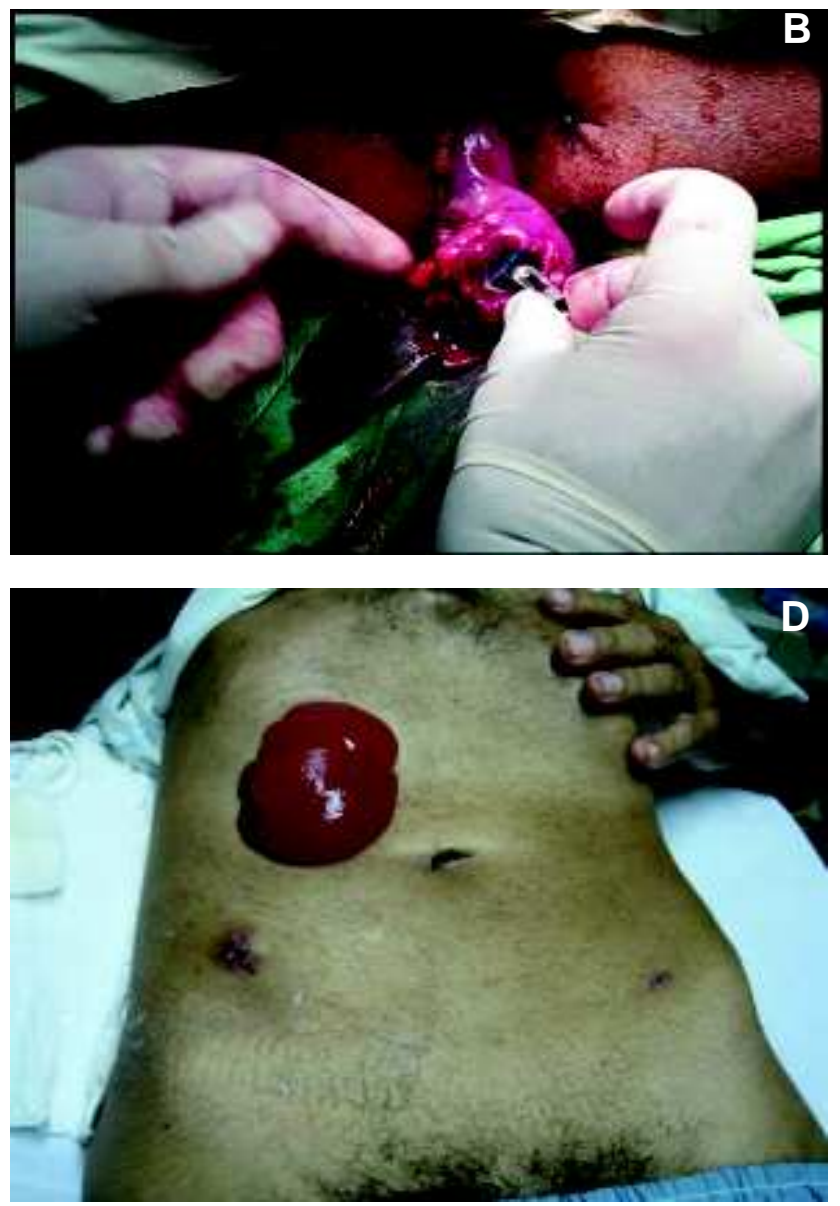
uma avaliação NOTES transgástrica utilizando endoscópio flexível demonstrou precisão em prever ressecabilidade semelhante à laparoscopia em 9 de 10 pacientes. A determinação da ressecabilidade foi decidida baseada em laparoscopia ou NOTES transgástrico por examinadores independentes e cegos para os achados do outro, e demonstrou factibilidade da exploração transgástrica flexível da cavidade abdominal. Em outras pequenas séries de uso de cirurgia por orifícios naturais para câncer, não foi registrada implantação tumoral nos acessos, mas sem dúvida é uma possibilidade em se tratando de ressecções oncológicas.

Embora vários investigadores tenham escolhido para pesquisa os acessos NOTES para a cavidade abdominal através de via transgástrica ou vaginal, poucos investigaram o acesso transcolônico ${ }^{3-8}$. A abordagem transcolônica tem várias vantagens teóricas sobre a via transgástrica, pela eliminação da necessidade de retroflexão do endoscópio, permitindo um acesso mais direto, além de o anoreto permitir a passagem de espécimes e instrumental de diâmetro maior que a via peroral ${ }^{37}$. A extração transcolônica ou transvaginal de espécimes em cirurgia laparoscópica tem sido mais utilizada nos últimos anos para permitir extração de baço, rins e cólon, eleiminando a necessidade de incisão auxiliar. Estas técnicas, classificadas como NOSE (natural orifice specimen extraction ${ }^{38}$ ) demonstraram bons resultados com poucas complicações relatadas 39-45. Colectomia NOTES Transvaginal assistida por minilaparoscopia foi iniccialmente descrita clinicamente por Lacy e cols, denominando a técnica de MANOS $^{46}$ em uma paciente com câncer de sigmóide. Burghardt e cols descreveram um caso de colectomia transvaginal híbrida com auxílio laparoscópico ${ }^{47}$.

NOTES transcolônico utilizando sistemas transanais rígidos foi objeto de pesquisa em recentes estudos experimentais e em cadáver. Whiteford e cols demonstraram a factibilidade de ressecção de retossigmóide com NOTES rígido em três cadáveres, utilizando TEM (transanal endoscopic microsurgery), possibilitando linfadenectomia em bloco e extração e anastomose transanal ${ }^{37}$. Os maiores obstáculos para a realização de colectomia transretal usando sistemas rígidos foi, em sua opinião, o ângulo agudo criado pela protuberância do promontório e o alcance limitado dos instrumentos. Sylla e cols realizaram com sucesso uma técnica utilizando ressecção colônica transanal rígida, por vezes com auxílio adicional de acesso transgástrico flexível para possibilitar mobilização do cólon, em um set de 14 experimentos em suíno sem observação de sobrevida e também em cadáver ${ }^{48}$. Leroy e cols descreveram uma técnica combinada utilizando acesso rígido e flexível transretal e transgástrico, possibilitando sigmoidectomia em modelo animal ${ }^{49}$. A técnica utilizando o conceito de T-NOTES (Totally NOTES) resultou em sobrevida em todos os 5 porcos, e nenhum sinal de peritonite ou abscesso intra-abdominal foi encontrado nem na laparoscopia, nem na laparotomia com sacrifício realizada no $14^{\circ}$ DPO. Os autoes ressalvam a possibilidade técnica de realizar cirurgia colorretal por orifícios naturais utilizando instrumentos disponíveis.

Transanally Endoscopic Microsurgery (TEM), desenvolvida e descrita por Buess e cols no início da década de 80 é atualmente um procedimento minimamente invasivo estabelecido e boa alternativa para a maioria das lesões benignas do reto e possivelmente para adenocarcinoma $\mathrm{T} 1$, e representa um pioneiro esforço no camp[o da cirurgia por orifícios naturais ${ }^{34,50}$. $\mathrm{O}$ conceito de instalar uma plataforma intra-retal para realizar cirurgia colorretal, atualmente possibilitando ressecções "em manga" e anastomoses término-terminais foi inicialmente idealizada para ressecção de tumores localizados abaixo da reflexão peritoneal do reto extraperitoneal, evitando perfuração para a cavidade peritoneal A ressecção de tumores no reto superior com entrada na cavidade e fechamento por sutura do defeito tem sido recentemente descrita, sem aumento de complicaçõespós-operatórias ${ }^{51}$.

Excisão mesorretal total (TME), juntamente com linfadenectomia em bloco com ligadura alta da artéria mesentérica inferior representa o atual tratamento oncológico padrão na ressecção curativa do adenocarcinoma de reto. Como descrito por Heald in 1982, um plano preciso de dissecção ("holy plane") possibilitou melhoria na ressecabilidade com redução da disseminação local, em especial na margem circunferencial, reduzindo a indicação para a ressecção abdomino-perineal ${ }^{\text {9-11 }}$. Como evidenciado no presente estudo, uma TME adequada com preservação do envelope mesorretal pode ser obtida através da dissecção NOTES flexível. O uso do acesso NOTES perirretal (PNA) foi realizada na direção oposta (da margem anal à junção retossigmóide) à utilizada em laparoscopia e cirurgia convencional, executada ao longo dos planos de fusão embriológica, iniciando a dissecção ao ganhar acesso ao plano pré-sacral localizado entre o mesorreto 
e a fascia pré-sacral. A entrada e a posterior evolução da dissecção por endoscópio flexível neste plano avascular foi mais difícil que na laparoscopia formal devido à inexistencia de instrumentos adequados, mas o retropneumoperitôneo melhorou a exposição e dissecção dentro do plano anatômico.

Naturalmente, o acesso transretal provoca dúvidas no tocante ao controle de contaminação e necessita do fechamento seguro do orifício criado ${ }^{8,52,53}$. Entretanto, um ponto de entrada baixo como descrito neste estudo e utilizando um túnel retroperitoneal ao invés de introdução pela junção reto-sigmóide, previamente fechando o reto proximal, permite a operação em um campo relativamente isolado que pode ser propriamente desinfectado, evitando preparo mecânico do cólon. Apesar da necessidade de uma perfuração retal para sua realização, escolhendo este ponto na exata linha de ressecção e anastomose planejada minimiza o temor de uma viscerotomia desnecessária. $\mathrm{O}$ acesso original NOTES transcolônico perirretal PNA oferece uma segura e baixa perfuração do reto, com simples e segura entrada e fechamento sob visualização direta. A insuflação retroperitoneal e retroperitoneoscopia tam- bém representa um acesso original para realizar biópsias de lesões e linfonodos retroperitoneais, e ulteriormente, possibilitar cirurgia NOTES pancreática, renal e adrenal. A abordagem PNA promove um rápido e simples acesso também à cavidade abdominal evitando a necessidade de tecnologias de alto custo. Em termos de filosofia cirúrgica, a cirurgia colorretal transcolônica parece fazer sentido, muito mais que a perfuração do cólon para realização de cirurgias de órgãos em outros sítios. A endoscopia flexível padrão e futuras plataformas flexíveis são indubitavelmente tecnologias adjuvantes para a realização de técnicas NOTES transcolônicas.

A nova técnica de NOTES perirretal PNA para o câncer retal adere aos princípios de ressecção oncológica e abre um amplo leque de possibilidades de aplicações futuras do NOTES transcolônico para a cirurgia colorretal. Apesar das vantagens sobre os métodos laparoscópico e convencional ainda não serem evidentes nesta experiência preliminar e lançamento deste conceito, a realização futura de cirurgias colorretais flexíveis transorificiais parece promissora.

\begin{abstract}
Objectives: Clinical natural orifice surgery has been applied for abdominal surgery in recent years. Despite potential advantages of transcolonic NOTES for colorectal diseases, it was since now not yet clinically applied. The study describes the first successful human application of transcolonic NOTES in the literature, in a new transrectal TME procedure for rectal cancer. Methods: IRB approval was obtained at the institution for the study, and the patient signed informed consent. In a 54yrs-old male patient with a rectal adenocarcinoma, total mesorectal resection and rectosigmoidectomy with lymphadenectomy was performed using a posterior transcolonic access $3 \mathrm{~cm}$ from the anal verge. Mesorectal dissection was achieved using a flexible colonoscope and endoscopic instrumentation and laparoscopic assistance. The specimen was extracted transanally, and transorificial anastomosis was performed, with proximal stoma. Results: Operative time was $350 \mathrm{~min}$, no intraoperative complications occurred. The postoperative course was uneventful, patient was discharged after 6 days. Conclusion: Successful first human report on Transcolonic NOTES potentially brings new fronteers and applications for minimally invasive surgery. The treatment of colorectal diseases through a flexible Perirectal NOTES Access (PNA) is a promising new approach besides existing laparoscopic and open surgery to improve patient care.
\end{abstract}

Key words: Rectal cancer; Total mesorectal excision; colorectal surgery; TME; Natural orifice surgery; NOTES; Transcolonic; Perirectal NOTES Access; PNA; Minimally invasive surgery.

\section{REFERÊNCIAS}

1. Sodergreen MH, Clarck J, Athanasiou T, Teare J, Yang GZ, Darzi A. Natural orifice translumenal endoscopic rurgery: critical appraisal of applications in clinical practice. Surg Endosc 2009; 23(4): 680-687.

2. Pasricha PJ, Krummel TM. NOTES and other emerging trends in gastrointestinal endoscopy and surgery: The change that we need and the change that is real. Am J Gastroenterol 2009; 104: 2384-2386.
3. Pai RD, Fong DG, Bundga ME, Odze RD, Rattner DW, Thompson CC. Transcolonic endoscopic cholecystectomy: a NOTES survival study in a porcine model (with video). Gastrointest Endosc 2006; 64:428-434.

4. Wilhelm D, Meining A, von Delius S, Fiolka A, Can S, von Weyhern CH, Schneider A, Feussner H. An innovative, safe and sterile sigmoid access (ISSA) for NOTES. Endoscopy 2007; 39: 401-406.

5. Ryou M, Fong DG, Pai RD, Sauer J, Thompson CC. Evaluation of a novel access and closure device for NOTES applications: 
a transcolonic survival study in the porcine model. Gastrointest Endosc 2008; 67(6) : 964-969.

6. Ryou M, Thompson CC. Techniques for transanal access to the peritoneal cavity. Gastrointest Endoscopy Clin N Am 2008; 18: 245-260.

7. Sporn E, Bachman SL, Miedema BW, Loy TS, Calaluce R, Thaler K. Endoscopic colotomy closure for natural orifice surgery using a T-fastener prototype in comparison to conventional laparoscopic suture closure. Gastrointest Endosc 2008; 68 (4): 724-730.

8. Bachman SL, Sporn S, Furrer JL, AstudilloJA, Calaluce R, McIntosh MA, Miedema BW, Thaler K.. Colonic sterilization for natural orifice translumenal endoscopic surgery (NOTES) procedures: a comparison of two decontamination protocols. Surg Endosc 2009; 23:1854-1859.

9. Heald RJ, Ryall RD.Recurrence and survival after total mesorectal excision for rectal cancer. Lancet. 1986 Jun 28;1(8496):1479-82.

10. Heald RJ, Husband EM, Ryall RD. The mesorectum in rectal cancer surgery: the clue to pelvic recurrence? Br J Surg 1982; 69:613-616.

11. Lujan J, Valero G, Hernandez Q, Sanchez A, Frutos MD, Parrilla P. Randomized clinical trial comparing laparoscopic and open surgery in patients with rectal cancer. Br J Surg. 2009 Sep;96(9):982-9.

12. Kalloo AN, Singh VK, Jagannath BS, Niiyama H, Hill SL, Vaughn CA, Magee CA, Kantsevoy SV. Flexible transgastric peritoneoscopy: a novel approach to diagnostic and therapeutic interventions in the peritoneal cavity. Gastrointest Endosc 2004; 60(1): 287-292.

13. Zorron R. Techniques of transvaginal access for NOTES. Tech Gastrointest Endosc 2009; 11: 75-83.

14. Zorron R, Filgueiras M, Maggioni LC, Pombo L, Carvalho GL, Oliveira AL. NOTES Transvaginal cholecystectomy: Report of the first case. Surg Innov 2007; 14(4): 279-283.

15. Marescaux J, Dallemagne B, Perretta S, Wattiez A, Mutter D, Coumaros D. Report of transluminal cholecystectomy in a human being. Arch Surg 2007; 142: 823-826.

16. Zornig C, Emmerman A, von Waldenfels HA, Mofid H. Laparoscopic cholecystectomy without visible scar: combined transvaginal and transumbilical approach. Endoscopy 2007; 39(10): 913-915.

17. Branco Filho AJ, Noda RW, Kondo W, Kawahara N, Rangel M, Branco AW. Initial experience with hybrid transvaginal cholecystectomy. Gastrointest Endosc. 2007; 66(6):12451248.

18. Ramos AC, Murakami A, Galvão Neto M, Galvão MS, Silva AC, Canseco EG, Moyses Y. NOTES Transvaginal videoassisted cholecystectomy: first series. Endoscopy 2008; 40(7):572-575.

19. DeCarli L, Zorron R, Branco A, Lima FC, Tang M, Pioneer SR, Sanseverino JI, Menguer R, Bigolin AV, Gagner M. New hybrid approach for NOTES transvaginal cholecystectomy:
Preliminary clinical experience. Surg Innov 2009; 16(20):181186.

20. Forgione A, Maggioni D, Sansonna F, Ferrari C, Di Lernia S, Citterio D, Magistro C, Frigerio L, Pugliese R. Transvaginal endoscopic cholecystectomy in human beings: preliminary results. J Laparoendosc Adv Surg Tech A. 2008; 18(3):345-351.

21. Zorron R, Maggioni LC, Pombo L, Oliveira AL, Carvalho GL, Filgueiras M. NOTES Transvaginal cholecystectomy: Preliminary clinical application. Surg Endosc 2008; 22(2): 542547.

22. Gumbs AA, Fowler D, Milone L, Evanko JC, Ude AO, Stevens P, Bessler M. Transvaginal natural endoscopic surgery cholecystectomy: early evolution of the technique. Ann Surg 2009; 249 (6): 908-912.

23. Horgan S, Cullen JP, Talamini MA, Mintz Y, Ferreres A, Jacobsen GR, Sandler B, Bosia J, Savides T, Easter DW, Savu MK, Ramamoorthy SL, Whitcomb E, Agarwal S, Lukacz E, Dominguez G, Ferraina P. Natural orifice surgery: initial clinical experience. Surg Endosc 2009; 23(7): 1512-1518.

24. Fischer LJ, Jacobsen G, Wong B, Thompson K, Bosia J, Talamini M, Horgan S. NOTES laparoscopic-assisted transvaginal sleeve gastrectomy in humans-description of preliminary experience in the United States. Surg Obes Relat Dis. 2009; 5(5):633-636.

25. Sousa LH, Sousa JAG, Sousa MM, Sousa VM, APM Sousa, Zorrón R.TOTALLY NOTE.S (T-NOTES) Transvaginal Cholecystectomy using Two Endoscopes: Preliminary report. Surg Endosc 2009; epub ahead of print. PMID: 19343424 [PubMed - as supplied by publisher]

26. Noguera J, Dolz C, Cuadrado A, Olea J, Vilella A, Morales R. Hybrid transvaginal cholecystectomy, NOTES, and minilaparoscopy: analysis of a prospective clinical series. Surg Endosc 2009; 23: 876-881.

27. Palanivelu C, Rajan PS, Rangarajan M, Prasad M, Kalyanakumari V, Parthasarathi R, Senthilnathan P. Transvaginal endoscopic cholecystectomy in humans: preliminary report of a case series. Am J Gastroenterol. 2009;104(4):843-847.

28. Davila F, Tsin DA, Dominguez G, Davila U, Jesús R, Gomez de Arteche A. Transvaginal cholecystectomy without abdominal ports. JSLS. 2009;13(2):213-216.

29. Rao GV, Reddy DN, Banerjee R. NOTES: Human Experience. Gastrintest Endoscopy Clin N Am 2008; 18: 361-370.

30. Marks JM, Ponsky JL, Pearl JP, McGee MF. PEG rescue: a practical NOTES technique. Surg Endosc. 2007; 21(5): 816819.

31. Palanivelu C, Rajan PS, Rangarajan M, Parthasarathi R, Senthilnathan P, Prasad M. Transvaginal endoscopic appendectomy in humans: a unique approach to NOTESworld's first report. Surg Endosc 2008; 22(5):1343-1347.

32. Ramos AC, Zundel N, Neto MG, Maalouf M. Human hybrid NOTES transvaginal sleeve gastrectomy: initial experience. Surg Obes Relat Dis. 2008;4(5):660-663. 
33. Seifert H, Wehrmann T, Schmit T, Zeuzem S, Caspary WF. Retroperitoneal endoscopic debridement for infected peripancreatic necrosis. Lancet 2000; 19(356): 653-655.

34. Buess G, Kipfmüller K, Ibald R, Heintz A, Hack D, Brausntein $\mathrm{S}$, Gabbert H. Junginger T. Clinical results of transanal endoscopic microsurgery. Surg Endosc 1988; 2 : 245-250.

35. Hazey JW, Narula VK, Renton DB, Reavis KM, Paul CM, Hinshaw KE, Muscarella P, Ellison EC, Melvin WS. Natural orifice transgastric endoscopic peritoneoscopy in humans: initial clinical trial. Surg Endosc 2008; 22: 16-20.

36. Zorron R, Soldan M, Filgueiras M, Maggioni LC, Pombo L, Oliveira AL. NOTES Transvaginal for cancer diagnostic staging: Preliminary clinical application. Surg Innov 2008 15(3):161165.

37. Whiteford M, Denk EM, Swanstrom L. Feasibility of radical sigmoid colectomy performed as natural orifice translumenal endoscopic surgery (NOTES) using transanal endoscopic microsurgery. Surg Endosc 2007; 21:1870-1874.

38. Palanivelu C, Rangarajan M, Jategaonkar PA, Anand NV. An innovative technique for colorectal specimen retrieval: a new era of "natural orifice specimen extraction" (N.O.S.E). Dis Colon Rectum. 2008; 51(7):1120-1124.

39. Delvaux G, Devroey P, De Waele B, Willems G. Transvaginal removal of gallbladders with large stones after laparoscopic cholecystectomy. Surg Laparosc Endosc 1993 3(4): 307-309.

40. Zornig C, Emmerman A, von Waldenfels HA, Felixmuller C. Colpotomy for specimen removal in laparoscopic surgery. Chirurg 1994; 65(10): 883-885.

41. Breda G, Silvestre P, Giunta A, Xausa D, Tamai A, Gherardi L. Laparoscopic nephrectomy with vaginal delivery of the intact kidney. Eur Urol 1993; 24(1):116-117.

42. Abrao MS, Sagae UE, Gonzales M, Podgaec S, DiasJr JA. Treatment of rectosigmoid endometriosis by laparoscopically assisted vaginal rectosigmoidectomy. Int J Gynaecol Obstet 2005; 91(1): 27-31.

43. Velhote MCP, Velhote CEP. A NOTES modification of the transanal pull-through. J Laparoendosc Adv Surg Tech 2009; 19(2): 255-257.

44. Franklin M, Kelley H, Kelley M, Brestan L, Portillo G, Torres J. Transvaginal extraction of the specimen after total laparoscopic right hemicolectomy with intracorporeal anastomosis. Surg Laparosc Endosc Percutan Tech 2008; 18 : 294-298.
45. Akamatsu H, Omori T, Oyama T, Tori M, Ueshima S, Nakahara M, Abe T, Nishida T. Totally laparoscopic sigmoid colectomy: a simple and safe technique for intracorporeal anastomosis. Surg Endosc 2009 Mar 6. [Epub ahead of print] PMID: 19266229

46. Lacy AM, Delgado S, Rojas OA, Almenara R, Blasi A, Llach J. MA-NOS radical sigmoidectomy: reporto f a transvaginal resection in the human. Surg Endosc 2008 Jul; 22(7): $1717-$ 1723.

47. Burghardt J, Federlein M, Müller V, Benhidjeb T, Elling D, Gellert K. Minimal invasive transvaginal right hemicolectomy: report of the first complex NOS (natural orifice surgery) bowels operation using a hybrid approach. Zentralbl Chir. 2008 Dec;133(6):574-6.

48. Sylla P, Willingham FF, Sohn DK, Gee D, Brugge WR, Rattner DW. NOTES rectosigmoid resection using transanal endoscopic microsurgery (TEM) with transgastric endoscopic assistance: a pilot study in swine. J Gastrointest Surg 2008;12(10):1717-23.

49. Leroy J, Cahill RA, Perretta S, Forgione A, Dallemagne B, Marescaux J. Natural orifice translumenal endoscopic surgery (NOTES) applied totally to sigmoidectomy: an original technique with survival in a porcine model. Surg Endosc 2009; 23:24-30.

50. Buess G, Becerra-Garcia F, Misra MC. Instruments for translumenal laparoscopic surgery or NOTES. Minim Invasive Ther Allied Technol 2008; 17 (6): 331-335.

51. Gavagan JA, Whiteford MH, Swanstrom LL (2004) Fullthickness intraperitoneal excision by transanal endoscopic microsurgery does not increase short-term complications. Am J Surg 187:630-634.

52. Rattner D, Kalloo, A. ASGE/SAGES Working Group on Natural Orifice Translumenal Endoscopic Surgery. Surg Endosc 2006; 20: 329-333.

53. Kantsevoy SV. Infection Prevention in NOTES. Gastrintest Endoscopy Clin N Am 2008; 18: 291-296.

\section{Endereço para correspondencia: PROF. RICARDO ZORRON}

Hospital Municipal Lourenço Jorge

University Hospital Teresopolis HCTCO

Rio de Janeiro - Brasil

E-mail: rzorron@terra.com.br 